\title{
Does PET/CT render software registration obsolete?
}

\section{U. Pietrzyk}

Institute of Medicine, Research Center Jülich, Brain Imaging Center West (BICW) and Department CPhysics, University of Wuppertal, Germany

\section{Keywords}

$\mathrm{PET} / \mathrm{CT}$, multi-modality image registration, image fusion

\section{Summary}

It was the success of software-based image registration that eventually led to the introduction of hardware-based concepts for image fusion, such as combined PET/CT tomographs. A prototype PET/CT was first presented in 1998, with various commercial designs to follow since 2000. PET/ CT is used primarily as a diagnostic modality in the field of extra-cerebral oncology imaging. The major advantage of combined imaging over retrospective software registration is the nearly identical position of the patient during both complementary examination, and therefore tomograms of identical parts of the body can be provided in spatially-corresponding slices.

Despite the availability of hardware combinations of complementary imaging modalities software-based image registration, however, still inherits a major role in subsequent data processing, in particular when individual imaging modalities other than combined PET/CT are being used during patient workup. Furthermore, software is likely to become an important tool for the correction of residual motion-induced mis-registration within combined PET/CT data sets, and for follow-up studies involving, for example, CT, PET, and PET/CT. Therefore, flexible algorithms that utilize nonlinear interpolation schemes implemented on fast computer systems are needed, and will continue to contribute to successful image registration and fusion in clinical practice.

Nuklearmedizin 2005; 44: S13-7

\section{Schlüsselwörter}

$\mathrm{PET} / \mathrm{CT}$, multimodale Bildregistrierung, Bildfusion

\section{Zusammenfassung}

Es war der Erfolg der Software-gestützten Bildregistrierung, der schließlich zur Einführung Hardware-gestützter Konzepte zur Bildfusion, wie z. B. des PET/CT, geführt hat. Ein Prototyp PET/CT wurde erstmalig 1998 vorgestellt, wonach seit 2000 weitere verschiedene kommerzielle Varianten entstanden. PET/CT wird primär zur Diagnostik auf dem Gebiet der extrazerebralen onkologischen Bildgebung eingesetzt. Der wichtigste Vorteil der kombinierten Bildgebung gegenüber retrospektiver Software-basierter Registrierung liegt in der nahezu identischen Position des Patienten während beider, sich ergänzenden Untersuchungen. Dadurch sind Daten identischer Teile des Körpers in räumlich sich entsprechenden Schnittbilder verfügbar.

Trotz der Verfügbarkeit Hardware-gestützter Kombinationen komplementärer Bild gebender Verfahren belegt die Software-gestützte Bildregistrierung weiterhin eine wichtige Rolle in der sich anschließenden Datenanalyse, insbesondere dann, wenn andere einzelnen Bild gebende Verfahren während anderer Untersuchungen des Patienten eingesetzt wurden. Ferner werden Software-Werkzeuge dann wichtig, wenn es darum geht, kleine, durch Bewegungen verursachte Fehlregistrierungen in den PET/CT Daten zU korrigieren und auch im Rahmen von Untersuchungen zur Verlaufskontrolle unter Einbezug von CT, PET und PET/CT. Flexible Algorithmen, die Ansätze der nicht-linearen Interpolation berücksichtigen und auf schnellen Computersystemen implementiert werden können, werden daher auch weiterhin zur erfolgreichen Bildregistrierung und Bildfusion besonders für die klinische Anwendung beitragen.

lower-resolution nuclear medicine images (PET or SPECT) with higher-resolution morphologic imaging (MRI or CT). Image registration and fusion were driven primarily by the field of neurology, to accurately localize activated cerebral areas during functional activation studies $(3,4)$. Thus, the first retrospective image registration algorithms in medicine were developed for research and clinical application in

\section{Wird Software-gestützte Bildregistrierung durch PET/CT überflüssig?}

the brain (5-7). Figure 1 shows a typical example of brain image registration.

In the following we will rehearse the challenges of image registration, in particular for extra-cerebral studies, and discuss survival strategies for software registration in the presence of combined imaging devices, such as PET/CT (8). In any case, retrospective image registration will be accepted in clinical practice only if it is based on robust, accurate and validated algorithms.

\section{Quest and solutions for image registration in brain studies}

Robustness and accuracy of software-based registration have been demonstrated for brain applications, whereby the brain can be considered a rigid organ (unlike extra-cerebral parts of the body). Figure 1 illustrates an example of PET-MRI image registration of the brain, which reflects a diagnostic strategy called "MRI-guided PET" (W.D. Heiss, 1990) (5). MRI-guided PET was pursued by the end of the 1980's in an attempt to support low-resolution PET and SPET brain images with anatomical background information. Of course, after defining a clinical motivation for retrospective image fusion, technical and methodological problems had to be solved.

By treating the brain (and head) as a rigid body, six degrees of freedom exist ( 3 for translation and 3 for rotation) to transform an image volume made up of stacks of 2D-images into the coordinate system of the other modality ( $(9-11)$ for further details and definitions). Linear, rigid image registration assumes that by definition there are no deviations from a rigid body behavior, i. e. all non-rigid effects are neglected. A more 


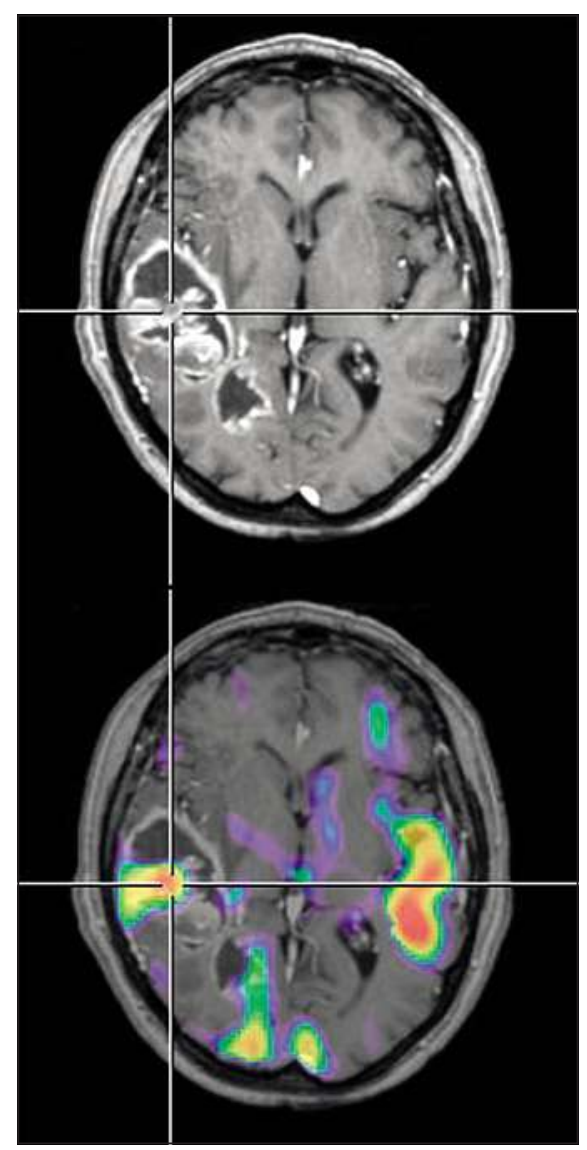

Fig. 1 Example of multimodality image registration and fusion (MRI, top; PET bottom) pre-surgical evaluation of a brain tumour. (Courtesy of A. Thiel and K. Herholz, Neurological Clinic, University of Cologne and Max-Planck Institute of Neurological Research, Cologne, Germany). general approach termed affine transformation also includes 3 parameters for scaling and 3 parameters for shears, thus, resulting in 12 degrees of freedom. Affine transformations may also account for slight differences in spatial calibration across scanners (e. g. pixel size and edge effects). There are a variety of applications for rigidbody image registration in brain studies, such as follow-up examinations of the same patient after therapy (12), or cognitive neuroscience studies of the network of activated brain regions in response to specific physical or mental tasks (13). Additional applications of brain image registration included image-guided biopsies and optimized surgical interventions. Table 1 provides an overview of transformation schemes frequently associated with specific clinical tasks involving the use of PET, CT or PET/CT.

In general, however, image registration in brain studies remained a research and was used selectively rather than for clinical routine. Furthermore, even in brain research the initial assumption of rigid body transformations is not always valid, as demonstrated by developments of software packages like SPM (Statistical Parametric Mapping) $(14,15)$. For the sake of providing a standard reference brain, which is obtained from averaging multiple brain studies or from a brain atlas (16), the algorithm had to

\begin{tabular}{|c|c|c|c|c|}
\hline \multirow[t]{2}{*}{ type } & \multirow[t]{2}{*}{ modality } & \multicolumn{2}{|c|}{ transformation } & \multirow[t]{2}{*}{ typical application } \\
\hline & & brain & whole-body & \\
\hline 1 & PET & $\begin{array}{l}\text { rigid/affine } \\
\text { rigid/affine } \\
\text { rigid/affine }\end{array}$ & $\begin{array}{l}\text { non-rigid } \\
\text { non-rigid } \\
\text { non-rigid }\end{array}$ & $\begin{array}{l}\text { follow-up studies with FDG-PET } \\
\text { dual-tracer studies with }{ }^{124} \text { and FDG } \\
\text { follow-up studies with different tracers }\end{array}$ \\
\hline 2 & CT & rigid/affine & non-rigid & $\begin{array}{l}\text { perfusion studies, liver studies with } \\
\text { multiphasic contrast agents (assuming } \\
\text { involuntary patient motion in between } \\
\text { frames) } \\
\text { follow-up studies after radiofrequency } \\
\text { ablation }\end{array}$ \\
\hline 3 & PET, CT & $\begin{array}{l}\text { rigid/affine } \\
\text { non-rigid } \\
\text { non-rigid }\end{array}$ & $\begin{array}{l}\text { non-rigid } \\
\text { non-rigid } \\
\text { non-rigid }\end{array}$ & $\begin{array}{l}\text { complementary diagnosis } \\
\text { dual-modality follow-up } \\
\text { dual-modality radiation therapy plan- } \\
\text { ning }\end{array}$ \\
\hline 4 & $\mathrm{PET} / \mathrm{CT}$ & $\begin{array}{l}\text { rigid/affine } \\
\text { non-rigid }\end{array}$ & $\begin{array}{l}\text { non-rigid } \\
\text { non-rigid }\end{array}$ & $\begin{array}{l}\text { correction of motion-induced artefacts } \\
\text { follow-up studies }\end{array}$ \\
\hline
\end{tabular}

Tab. 1

Categories of image transformations associated with selected clinical tasks involving the use of PET, CT and PET/CT. be extended to non-rigid transformations to account for deviations between the individual brains and the reference brain. Nevertheless, deviations from the rigid brain model are by far easier to account for than corresponding deviations in extra-cranial studies, which can be generated by respiration and cardiac motion.

In summary, for brain imaging reliable retrospective image registration algorithms exist that are based on rigid (or non-rigid), frequently fully-automated, registration algorithms $(17,18)$, that are available either as research or commercial packages. However, despite the clinical success of brain registration in selected areas, the definition of quality standards and validation procedures of retrospective software registration remains an open issue.

\section{Failure of software based regis- tration in extra-cranial studies}

Software algorithms that were developed for brain applications cannot be transformed simply to extra-cranial applications. The major algorithmic challenge is that in contrast to the brain, all relevant extra-cranial parts of the body cannot be considered a rigid body.

Typically, complementary images differ as they are acquired either during different respiratory phases, or during several respiratory cycles, as depicted in Figure 2. Additional challenges arise when the examinations are not performed on the same day and when patients have to be transported to a remote site for further imaging.

With these limitations in mind, independently-acquired image volumes can be submitted to an image analysis chain including reformatting, transformation and interpolation, but they cannot be regarded to represent image information from rigid bodies anymore. Initially, software developers applied rigid-body transformations to only a limited range of continuous 2D-images (19). Anatomical and external landmarks have been used to guide the registration process (2). However, despite these, sometimes time-consuming attempts, there has not been any clinically-acceptable solution to retrospective software registration that is 
applicable to whole-body images and addresses all the requirements mentioned above.

\section{PET/CT - a powerful solution to the extra-cerebral image registration?}

There are two arguments, why a combined imaging device like PET/CT bears a significant advantage for routine image registration. By positioning the patient on a single, common table platform in front of the combined tomograph, misalignment from repositioning the patient can be excluded for the entire whole-body imaging range. This also eliminates the challenge of different patient positioning on different tomographs that retrospective algorithms faces due to differences in the curvature of the table pallets. While the assumption of a rigid body transformation is still not true for whole-body PET/CT tomography, the problem of non-linearity is confined intrinsically to limited axial ranges, such as the heart or the liver.

The advent of PET/CT developments came about with the recognition of the diagnostic potential from co-registered anatomical and functional images, as they were provided by initial software-based attempts. However, it was also the failure of the very algorithms in extra-cerebral studies that prompted physicists to design and construct a hardware-based solution to multi-modality imaging to overcome most of the limitations inherent to software-based image fusion.

With the advent of combined image registration through PET/CT, one might be tempted to argue, that software-based solutions are obsolete now. Indeed, PET/CT is not challenged by obstacles that are inherent to software-based image registration. For example, PET and CT images are acquired with the patient being positioned on a single patient support for the duration of the entire exam. Following both scans, the PET images are being corrected for attenuation using the available $\mathrm{CT}$ transmission images, and both images are ready to be viewed on a

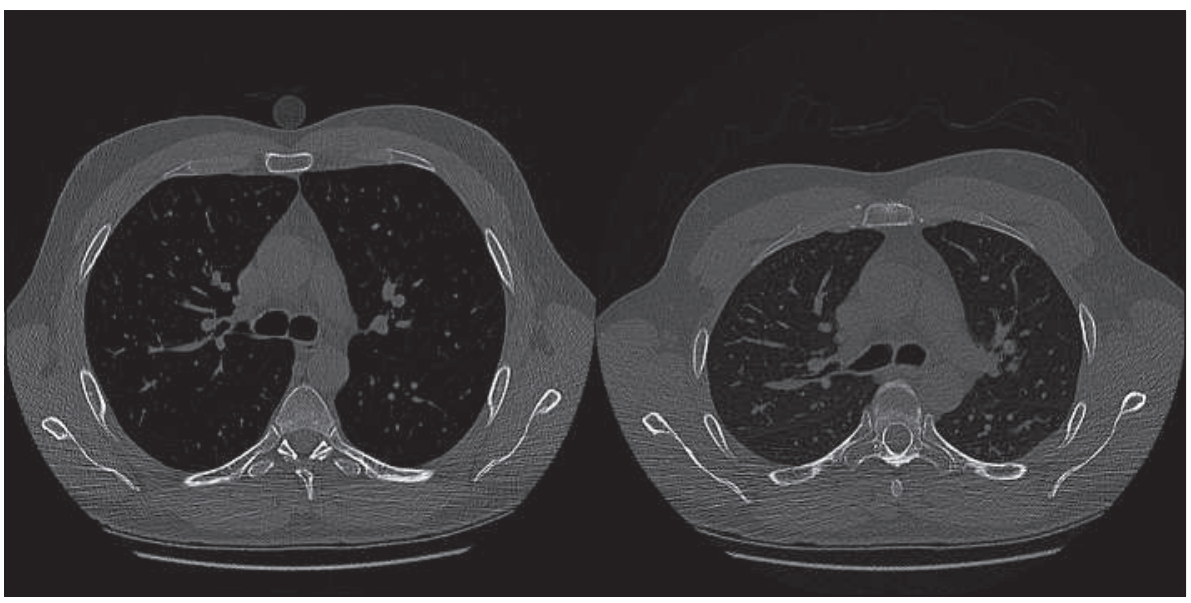

Fig. 2 CT-images acquired during different phases of the respiratory cycle (left: inhalation; right: exhalation). Obviously, differences in the anatomy are not confined to shifts in the axial image planes but also extent into neighbouring image planes.

single display shortly after the completion of the combined exam. Hence, PET/CT can be regarded as a single, fully-integrated imaging device.

The basic assumption for PET/CT, however, is that no relative movement of the patient occurs in between or during the two exam portions. Usually, the $\mathrm{CT}$ is performed first with the patient breathing quietly or during breath-hold in normal expiration, followed by the PET examination with the patient breathing quietly. Both parts of the examination involve different timeframes, with $\mathrm{CT}$ images being acquired in less than a minute, while average PET acquisition time is about in 3-4 min, or more per bed position, of which several are scanned to cover the axial imaging range. A typical pitfall for PET/CT is illustrated in Figure 3 where PET and CT are locally misaligned because of involuntary head motion arising from muscle relaxation in the neck between the two exams. Similar examples will serve as arguments against PET/CT for as long as the two complementary scans are not acquired simultaneously. There are, of course, other sources of motion-induced local misregistration, such as respiration and cardiac motion, which can be addressed in part by prospective measures as discussed by Schäfers and Nekolla in this supplement.

\section{Is software-based image registration still needed?}

Despite local mis-registration that may arise from involuntary patient motion, combined PET/CT represent the best intrinsic co-registration of multi-modality images available for clinical use today. This, and other potential hardware combinations, can also be regarded as the best starting point for software registration as an integrated option to multimodality hardware-based imaging.
Fig. 3

An example of a local, motion-induced mismatch between CT and PET data from combined a PET/CT scanner. Mis-registrations like the one displayed are seen frequently in PET/CT when the time delay in covering corresponding anatomical areas with $\mathrm{CT}$ and PET (in opposite scan directions) is $30 \mathrm{~min}$ or more.

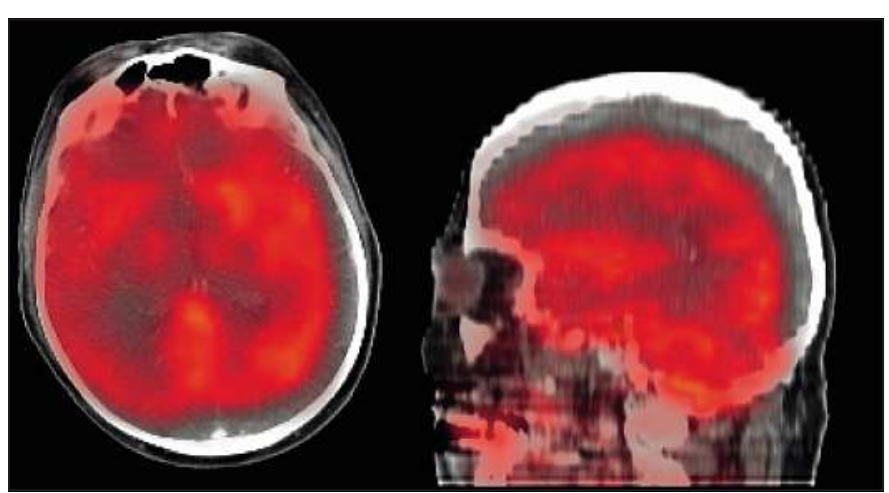




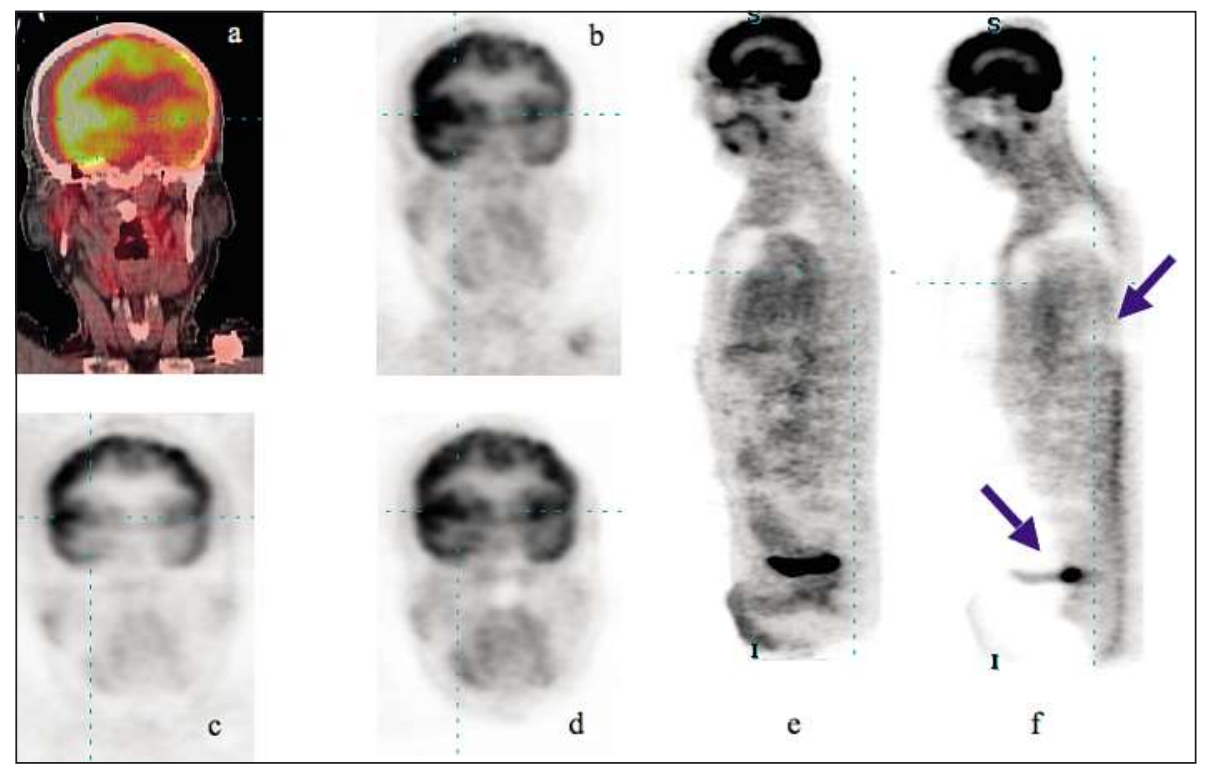

Fig. 4 Local motion-induced PET-CT misalignment in PET/CT studies may lead to a biased tracer distribution following CTbased attenuation correction.

a) fusion of the misaligned PET and CT images;

b) asymmetric FDG metabolism in the brain on PET image after aftenuation correction based on the original CT

c) symmetric FDG metabolism on PET image prior to attenuation correction

d) PET image following CT-based attenuation correction with realigned CT images.

Retrospective image registration - aimed at aligning the head - result in distant misalignment and artifacts (arrows) in the thorax and pelvis (f) compared to the original whole-body PET (e).

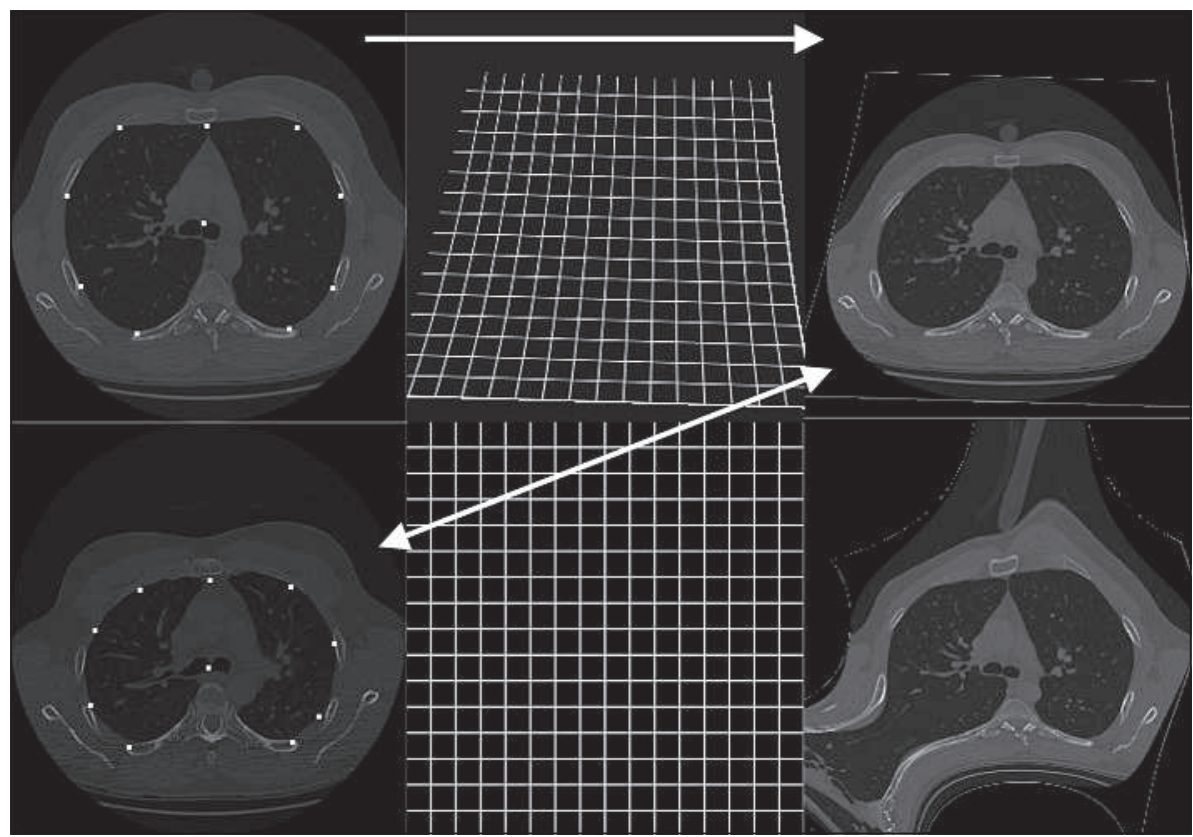

Fig. 5 Example strategy to warp a CT image acquired during a particular respiration phase (inhalation, top left) resulting in an image (top right), which is similar to the image from the exhalation phase (bottom left). The resulting transformation (middle; top), which is based on the landmarks shown in both image at the left side, deviates from the regular grid (middle; bottom). The selection of landmarks requires great care in order to avoid unstable solutions (bottom right).
One such add-on functionality could be a software utility, which corrects for motioninduced displacements of PET/CT data, such as in the example given in Figure 3. Some tools that are available today have rigid and non-rigid transformations available (see for example: MiraView from Mirada Solutions and Siemens Molecular Imaging) and (18). None of these commercial algorithms, however, is able to solve the general problem of image co-registration for image volumes covering more extensive parts of the body, or the entire whole-body imaging range. This is exemplified in Figure 4. Correcting for local motion-induced misalignment must start with the retrospective co-registration of the CT images to the uncorrected emission data (assuming no significant misalignment has occurred during the emission scan) before employing the coregistered CT in CT-based attenuation correction. However, using linear registration image artifacts in distant areas of axially extended image volumes may be expected (20).

Clinically acceptable solutions to image co-registration, however, require that no additional work-load is imposed onto the staff. Therefore, motion-correction schemes driven by manual landmark selection (Figure 5) appear inappropriate for future routine applications. The example in Figure 5 also demonstrates the challenges of finding a stable solution to the non-rigid registration task. Thus, focusing on a limited axial range, or the local area of misalignment, may perhaps be a pre-requisite for future developments of software-based correction schemes of PET/CT data.

An open question today, particularly for non-rigid image registration, is the need for a standard reference and quality measures, which can be used to control, validate and evaluate these software-based solutions. In other words, new technologies should be robust, accurate and validated and be operated without the need of user interaction. It is this constraint, which makes software-based image registration alone a challenging task. Although Kim and colleagues have concluded in a recent study that "software fusion currently cannot be considered to represent a valid alternative to in-line PET/ CT" (21), we see new avenues for improved 
$\mathrm{PET} / \mathrm{CT}$ and software registration

and validated software registration tools that may contribute to make PET/CT a quality procedure in clinical routine with new applications exceeding that of simple one-time imaging.

\section{Conclusion}

PET/CT provides an advanced and improved starting point for software developers. Nevertheless, essential tools are still needed in the presence of PET/CT, such as tools to align multiple studies for therapy follow-up assessment and for correcting residual motion-induced mis-registration. For extra-cranial imaging, PET/CT today is definitely the best approach that can provide accurately aligned images. However, despite the unprecedented success of PET/CT hardware fusion software solutions are not obsolete yet.

\section{Acknowledgement}

Part of this work was supported by a grant by the DFG BE 3169/2-2.

\section{References}

1. Kuhl DE, Hale J, Eaton WL. Transmission scanning: a useful adjunct to conventional emission scanning for accurately keying isotope deposition to radiographic anatomy. Radiology 1966; 87: 278-84.
2. Wahl RL, Quint LE Cieslak RD et al. Anatometabilic tumor imaging: fusion of FDG PET with CT and MRI to localize foci of increased activity. J Nucl Med 1993; 34: 1190-7.

3. Herholz K, Thiel A, Wienhard K et al. Individual functional anatomy of verb generation. Neuroimage 1996; 3: 185-94.

4. Thiel A, Herholz K, von Stockhausen H-M et al. Localization of language-related cortex with ${ }^{15} \mathrm{O}$-labeled water PET in patients with gliomas. Neuroimage 1998, 7: 284-95.

5. Pietrzyk U, Herholz K, Heiss W-D. Three-dimensional alignment of functional and morphological tomograms. Journal of Computer Assisted Tomography 1990; 14: 51-9.

6. Levin DN, Pelizzari CA, Chen GTY et al. Retrospective geometric correlation of MR, CT and PET images. Radiology 1988; 169 (817): 823.

7. Woods RP, Mazziotta JC, Cherry SR. MRI-PET registration with automated algorithm. Journal of Computer Assisted Tomography 1993; 17: $536-46$.

8. Townsend DW, Cherry SR. Combining anatomy and function: the path to true image fusion. Eur Radiol 2001; 11: 1968-74.

9. Hajnal JV, Hill DLG, Hawkes DJ (eds). Medical Image Registration. CRC Press 2001.

10. Hill DLG, Batchelor PG, Holden M et al. Medical Image Registration. Phys Med Biol 2001; 46: $1-45$.

11. Hutton BF, Braun M, Thurfjell L et al. Image registration: an essential tool for nuclear medicine Eur J Nucl Med 2002; 29: 559-77.

12. Thiel A, Herholz K, Koyuncu A et al. Plasticity of language networks in patients with brain tumors: a positron emission tomography activation study. Ann Neurol 2001; 50: 620-9.

13. Fink GR, Frackowiak RSJ, Pietrzyk U et al. Multiple non-primary motor areas in the human cortex. J Neurophysiol 1997; 77: 2164-74.
14. Friston KJ, Ashburner J, Frith CD et al. Spatial registration and normalization of images. Human Brain Mapping 1995; 2: 165-89.

15. www.fil.ion.ucl.ac.uk/spm/

16. Collins DL, Neelin P, Peters TM et al. Automatic 3D intersubject registration of MR volumetric data in standardized Talairach space. Journal of Computer Assisted Tomography 1994; 18: 92-205.

17. Maes F, Collignon A, Vandermeulen D et al. Multimodality image registration by maximization of mutual information. IEEE Transactions on Medical Imaging 1997; 16: 187-98.

18. Crum WR, Hartkens T, Hill DLG. Non-rigid image registration: Theory and practice. $\mathrm{Br} \mathrm{J}$ Radiol 2004; 77: S140-53.

19. Slomka PJ, Dey D, Przetak C et al. Automated 3-dimensional registration of stand-alone ${ }^{18}$ F-FDG whole-body PET and CT. J Nucl Med 2003; 44: 1156-67.

20. Beyer T, Bruckbauer T, Howe E et al. Combining hardware and software PET/CT image registration for improved dual-modality oncology imaging. J Nucl Med 2005; 46: 462P.

21. Kim JH, Czernin J, Allen-Auerbach MS et al. Comparison between ${ }^{18} \mathrm{~F}$-FDG PET, PET/CT, and software fusion for restaging of recurrent colorectal cancer. J Nucl Med 2005; 46: 587-95.

Correspondence to:

Prof. Dr. Uwe Pietrzyk

Forschungszentrum Jülich $\mathrm{GmbH}$

Institut für Medizin

52425 Jülich

Tel. +49/(0)24 61/61 4763

Fax +49/(0)24 61/61 $2820+8229$

E-mail: U.Pietrzyk@fz-juelich.de 